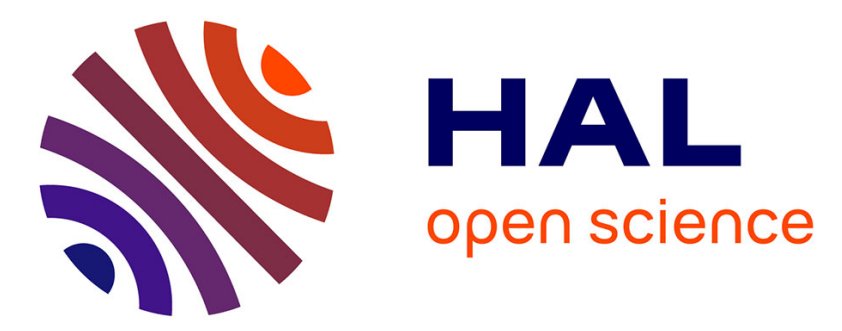

\title{
Geometry as a Universal Mental Construction
}

Véronique Izard, Pierre Pica, Stanislas Dehaene, Danièlle Hinchey, Elizabeth Spelke

\section{To cite this version:}

Véronique Izard, Pierre Pica, Stanislas Dehaene, Danièlle Hinchey, Elizabeth Spelke. Geometry as a Universal Mental Construction. Stanislas Dehaene; Elizabeth Brannon. Space, Time and Number in the Brain, Elsevier, pp.319-332, 2011, Attention and Performance series, 10.1016/B978-0-12-3859488.00019-0 . halshs-00603494

\section{HAL Id: halshs-00603494 https://shs.hal.science/halshs-00603494}

Submitted on 3 Apr 2017

HAL is a multi-disciplinary open access archive for the deposit and dissemination of scientific research documents, whether they are published or not. The documents may come from teaching and research institutions in France or abroad, or from public or private research centers.
L'archive ouverte pluridisciplinaire HAL, est destinée au dépôt et à la diffusion de documents scientifiques de niveau recherche, publiés ou non, émanant des établissements d'enseignement et de recherche français ou étrangers, des laboratoires publics ou privés. 


\title{
Geometry as a Universal Mental Construction
}

\author{
Véronique Izard ${ }^{* \dagger}$, Pierre Pica ${ }^{\ddagger}$, \\ Stanislas Dehaene $e^{\S \mathbb{I}^{* * \dagger}}$, Danielle Hinchey ${ }^{* * *}$, \\ Elizabeth Spelke $e^{* * *}$
}

"Laboratoire Psychologie de la Perception, Université Paris Descartes, Paris, France; ${ }^{\dagger}$ CNRS UMR 8158, Paris, France; ${ }^{\dagger}$ UMR 7023 "Formal Structure of Language", CNRS and Université Paris 8, Paris, France; ${ }^{\S}$ INSERM, Cognitive

Neuroimaging Unit, Gif-sur-Yvette, France; ${ }^{\mathbb{I}}$ CEA, I2BM, NeuroSpin, Gif-sur-Yvette, France; **:Université Paris-Sud, Orsay, France; ${ }^{\dagger \dagger}$ Collège de France, Paris, France; *** Department of Psychology, Harvard University, Cambridge, USA

\section{Summary}

Geometry, etymologically the "science of measuring the Earth", is a mathematical formalization of space. Just as formal concepts of number may be rooted in an evolutionary ancient system for perceiving numerical quantity, the fathers of geometry may have been inspired by their perception of space. Is the spatial content of formal Euclidean geometry universally present in the way humans perceive space, or is Euclidean geometry a mental construction, specific to those who have received appropriate instruction? The spatial content of the formal theories of geometry may depart from spatial perception for two reasons: first, because in geometry, only some of the features of spatial figures are theoretically relevant; and second, because some geometric concepts go beyond any possible perceptual experience. Focusing in turn on these two aspects of geometry, we will present several lines of research on US adults and children from the age of three years, and participants from an Amazonian culture, the Mundurucu. Almost all the aspects of geometry tested proved to be shared between these two cultures. Nevertheless, some aspects involve a process of mental construction where explicit instruction seem to play a role in the US, but that can still take place in the absence of instruction in geometry. 
The axioms of geometry introduced by Euclid circa 300 B.C. [1] define concepts with spatial content, such that any theorem or demonstration of Euclidean geometry can be realized in the construction of a figure. Just as intuitions about numerosity may have inspired the early mathematicians to develop mathematical theories of number and arithmetic, Euclid may have appealed to universal intuitions of space when constructing his theory of geometry. In the current chapter, we investigate this proposition by assessing how much of the spatial content of Euclidean geometry is present in our spontaneous intuitions about space.

There are two major aspects of the spatial content of Euclidean geometry that may depart from our perception of space. First, in geometry, only some of the features of spatial figures are theoretically relevant. For example, Euclid introduces axioms pertaining to angles, where he points to the right angle as a special figure; but he does not introduce definitions related to orientation, such as a definition of horizontal or vertical lines. In this sense, geometric representations may be more specific than spatial representations: to qualify as "geometric", spatial representations must instantiate invariance by the properties that are not theoretically relevant to formal geometry. Second, some of the concepts of geometry transcend spatial perception by their very definition. Hence, Euclid's axioms introduce ideal concepts whose extension in space is either infinitely small or large, extending beyond the limits of our perception. For example, for Euclid a line is an object so infinitely thin that it has no width, while at the same time its extension in the length direction may be infinite.

In the following, we will focus in turn on those two aspects of geometry and raise the questions of their universality and development. A first possibility would be that all humans have access to the spatial content of Euclidean geometry, either because we all come to learn

\section{BOX 19.1}

\section{TWO DISSOCIATED SYSTEMS OF CORE KNOWLEDGE FOR GEOMETRY}

A large part of the research effort in geometry has focused on navigation tasks, following the classic behavioral and neurophysiological research of Tolman [33] and O'Keefe and Nadel [34], respectively, and invigorated by Cheng's seminal discovery that animals use the geometry of their surroundings to establish their orientation [35]. The system encoding space for reorientation is truly geometric in the sense that it encodes information about the shape of the environment, and ignores featural information such as colors, or landmark objects [36] (but see [37] for a different view). However, the system of geometry-informed navigation fails to reach abstraction in two points. First, it fails to encode some geometric information, namely angle $[31,38,39]$. Second, it also fails to recognize geometry in $2 \mathrm{D}$ displays, a main domain of Euclidean geometry [31,40]. Nevertheless, sensitivity to $2 \mathrm{D}$ shapes is present even in infants [41], which leads us to postulate the existence of a second cognitive system of geometric content, dedicated to small, manipulable objects and 2D displays [31]. As reviewed in the present chapter, this system is sensitive to angle and length, while it ignores distinctions of sense: it is thus complementary to the geometry-informed navigation system, which encodes length and sense, but not angle. Children may need to learn to combine the information given by these two systems to create an integrated representation of Euclidean geometry. 
it on the basis of an experience of space that is general enough to be universal, or because Euclidean geometry expresses core aspects of our perception of space (Box 19.1). On the contrary, geometric concepts may only be available to those who have received relevant instruction, or invested considerable energy in their mental construction. To address these issues, the present chapter will present several lines of evidence involving children of different ages, and people from an Amazonian culture, the Mundurucu.

\section{UNIVERSAL GEOMETRIC INTUITIONS}

In a first study, we probed a variety of geometric intuitions in a people from the Amazon, the Mundurucu [2]. The participants had no instruction in geometry, and their language does not have terms for basic Euclidean concepts such as parallelism or right angle. In one test, participants had to detect an image that was "different" or "weird" in slides of six images. Five of these images illustrated a geometric property (e.g., parallelism), while the remaining one lacked this property (non-parallel lines) (Fig. 19.1A). Care was taken to introduce maximal variation on irrelevant aspects of the images. For instance, in the previous example, the distractor pair of lines varied in terms of the distance between the two lines, the length of each line, and their orientation. This variation created several options for the participants, who may have relied either on geometry or on other aspects of the shapes to elect their answer. Nevertheless, across a variety of trials targeting different geometric properties, adult and children Mundurucu used principally the abstract geometric properties of the figures and performed well above chance. Furthermore, their performance across trials correlated tightly with the performance of adult and children control participants from the US (Fig. 19.1B). Despite dramatic differences in geometric education between these two groups, the trials that were harder for the Munducuru were also harder for the US participants. This test therefore reveals a signature of geometric intuitions, by establishing a hierarchy of saliency between the different geometric and non-geometric properties of images. This signature is impervious to instruction in geometry, and potentially universal across cultures. More recently, we also found evidence for continuity through development in a study including 448 participants from the US, ranging in age from three to 51 years: the same correlations were observed across all age groups [3] (Fig. 19.1C).

However, because the test spans a large range of geometric properties, and each trial is unique, it cannot suffice to infer the content of our geometric intuitions. In particular, the correlational findings suggest that participants relied mostly on geometry in processing shapes, but also used non-geometric cues when choosing an outlying figure. In an attempt to better characterize the geometric content of our spatial intuitions, the following sections will present new experimental tests controlling more closely the type of spatial variations introduced in the images (geometric and non-geometric). These tests will focus on features that are particularly diagnostic of the concepts of Euclidean geometry: global size, length proportions, angle, sense, and orientation.

\section{PERCEPTION OF ABSTRACT GEOMETRIC FEATURES}

Under the framework of transformational geometry (Box 19.2), Euclidean geometry can be conceived as a list of embedded theories, which differ by the type of features they make 
(A) Examples of trials

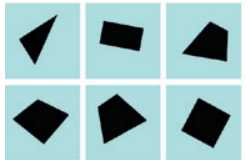

Triangle



Parallel lines

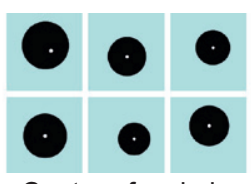

Center of a circle

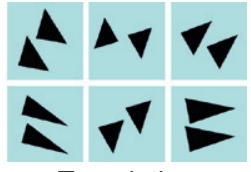

Translation

(B) Correlations of responses between Mundurucu and U.S. participants
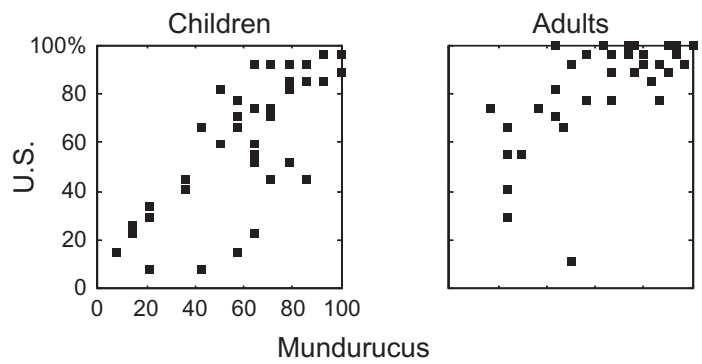

(C) Correlations of responses between U.S. children and adults

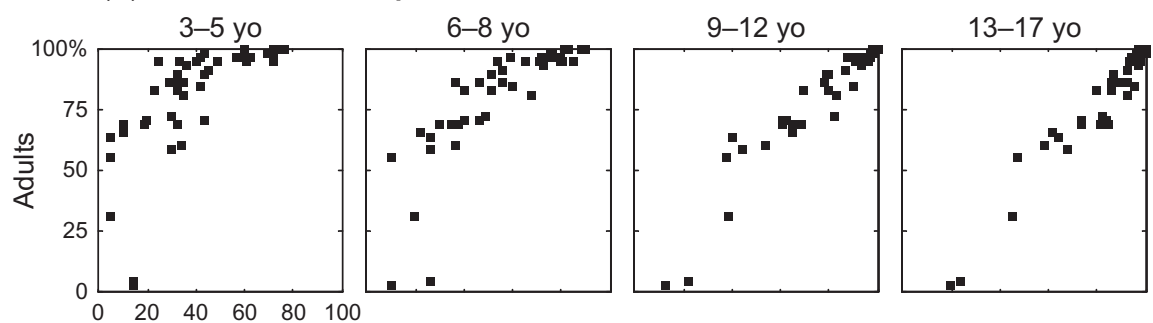

FIGURE 19.1 Generic test of geometric intuitions. Participants were presented with a series of 45 slides such as those in (A), each illustrating a distinct geometric property. In each slide, five of the images share a geometric property which the last image lacks. The participants were asked to pick the image that was "different", or "weird and ugly". The test was first administered to 14 children and 30 adults from the Mundurucu population, as well as control groups of 26 children and 28 adults in the US. The responses across trials were highly correlated between these two populations, in each age group (B). Later, the test was also administered to 448 participants from the US aged three to 51 years, and revealed the same correlation across age groups (C). A and B adapted from [2]; C adapted from [3].

explicit. Briefly, in all versions of Euclidean theory, angle and length proportions are defining features of figures, while position or orientation are not; the status of sense and global size is variable. From a psychologist's point of view, the framework of transformational geometry defines a research program: to look at the perception of some abstract geometric feature despite variations of other aspects of the displays, such as orientation, position, or global size [4]. If people are sensitive to geometry in the sense of one of the Euclidean theories, the defining features of this geometry should be easy to perceive, even with concomitant variations of non-defining features, and hard to ignore when their variation is made 


\section{BOX 19.2}

\section{TRANSFORMATIONAL GEOMETRY}

In Klein's transformational geometry framework, any geometric theory (such as Euclidean geometry) can entirely be defined by the set of its invariant transformations, i.e. transformations that do not affect the theorems of that theory [42]. For example, if a geometry theory is invariant by translation, then for that geometry two figures that are identical, except that they are placed in different positions in space, are considered equivalent: exactly the same list of theorems can be proven of either of these two figures. (Of course, the relationships between these two figures could be the object of other theorems, such as theorems describing how the sides of these two figures are parallel to each other: such theorems are concerned with the global figure formed of the two translated versions, and therefore do not consider the two subfigures equivalent. In other words, invariant transformations must be thought as irrelevant when applied to the whole figure, not only a subpart.)

The transformational framework allows us to think of geometric theories as embedded within each other, with increasing levels of invariance: as long as a subset of the parent geometry's invariant transformations set verifies certain combination properties, this subset defines a valid theory of geometry. For example, in 2D geometry, Euclid's axioms create a high level of invariance, with four types of invariant transformations: translation (position in space), rotation (orientation), symmetry (sense), and homothecy (global size) (see Box 19.2 Fig. 1). This list of invariant transformations leaves angle and length proportions as essential properties of a figure. By comparison, in the geometry created by solid movement of objects, which is embedded within Euclid's full theory, only translations and rotations are included in the set of invariant transformations: besides angle and length proportions, sense and global size are also defining features of figures in this geometry. Embedded between these two geometries, one can also define a Euclidean geometry that is sensitive to angle, length proportions, and global size while being agnostic to sense: we will refer to this system of geometry as "the geometry of non-oriented solid movements".



BOX 19.2 FIG. 1 Illustration of the four types of invariant transformations for Euclidean geometry. 
irrelevant to a task. On the contrary, the properties that are irrelevant to Euclidean geometry, such as orientation and position, should be easy to ignore. ${ }^{1}$

To our knowledge, the question has rarely been addressed in this form in the literature (see [8-11] for a few exceptions). However, evidence from infants [12-14], young children $[15,16]$, adults $[7-11,17,18]$, and single-unit recordings [19] (see a review of mirror image confusions in [20]) seem to indicate that length (both relative and absolute) and angle have a privileged status in form perception. In contrast, the perception of sense requires an additional step of mentally aligning the objects, as in the classical experiments of mental rotation [7]. This would argue that the intuitive basis for geometry is a non-oriented Euclidean geometry. However, all the evidence summarized above comes from a variety of tasks, displays, measures, and population, thus making direct comparisons difficult.

We designed a new test focusing specifically and systematically on the defining features of some or all of the Euclidean geometries: angle, size, and sense [3]. As in the original intruder test [2], participants were presented with six figures for each trial, and instructed to find the figure that was "very different". Each figure was shaped like an L, except that the orientation of the figure, its size, its sense (akin to an L or the mirror image of an L), and the angle between the two branches could vary. On "pure trials", one of the figures differed from the others in terms of size, angle, or sense, while all other parameters were kept constant, except for the global orientation and position of the figure (Fig. 19.2A). On "interference trials", a second dimension was allowed to vary progressively across figures, but not in a way that defined a unique deviant: for example, when size was the interfering dimension, each of the six figures was presented in a different size. The test was administered to 104 participants from three to 34 years of age. In all the groups tested, angle and size deviants were detected better than the sense deviants (Fig. 19.2B). Even the youngest children (mean age 3.91 years) were able to use angle and size, but only adults relied on sense in their search for the deviant figure. Furthermore, in "interference" trials, introducing irrelevant variations of size or angle impaired the detection of the deviant, while participants were not disturbed by irrelevant variations of sense (Fig. 19.2C). In fact, when an irrelevant variation of size or angle was present, participants sometimes used this dimension to make their choice, electing for example the largest or the smallest shape.

More recently, we observed the same pattern of performance in a group of 25 Mundurucu participants: on the "pure" trials, adults and children detected the size and angle deviants successfully while failing to detect the sense deviants. Similarly, "interference trials" revealed that they were sensitive to irrelevant variations of size and angle, but not sense.

Our results on sense may seem surprising, given that even preschoolers can succeed at mental rotation tasks, when they are given explicit instruction or when the stimuli are embedded in an ecological Tetris game task [21,22]. In adults, the detection of sense variations is specific in that it requires the use of mental rotation, whereas metric differences can be perceived

\footnotetext{
${ }^{1}$ In terms of mechanisms, for any of the relevant features, organisms could opt for either of two computational solutions: the first solution would be to extract that feature directly, abstracting away non-relevant aspects in the figure. It has been proposed that this kind of solution is used for encoding numerosity [5], and reflects the organization of the visual system at large [6]. Another solution would be to impose a mental transformation and realign the displays, to then apply direct pattern matching between the realigned displays. This solution is used, for example, to detect sense deviations in classic mental rotation tasks [7].
} 
(A) Examples of trials Angle



angle, size interference

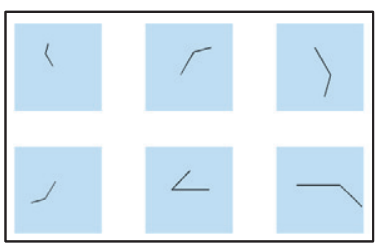

angle, sense interference

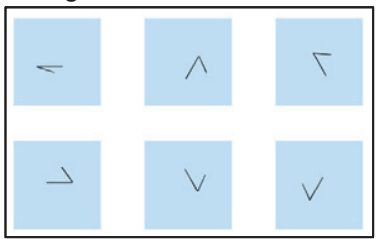

(B) Pure trials accuracy

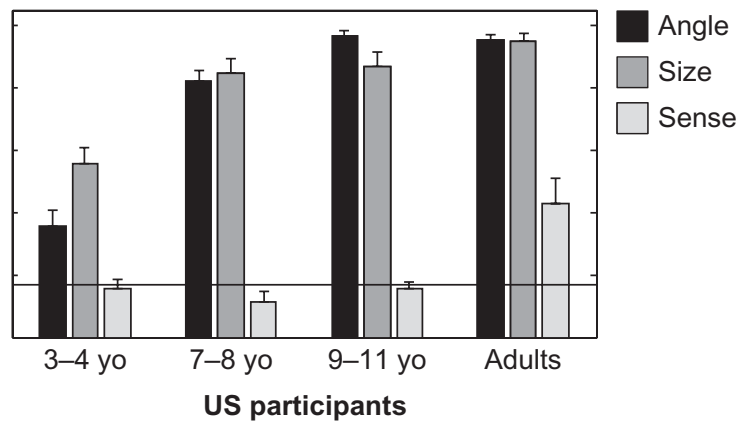

Pure trials
Size

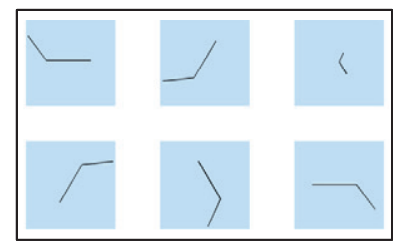

Interference trials

size, angle interference



size, sense interference

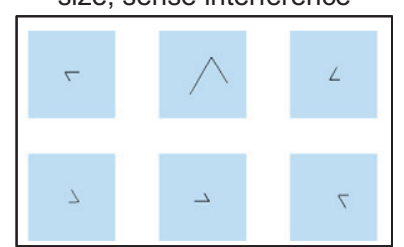

Sense

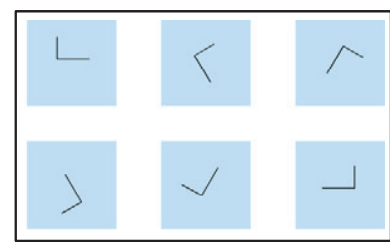

sense, angle interference

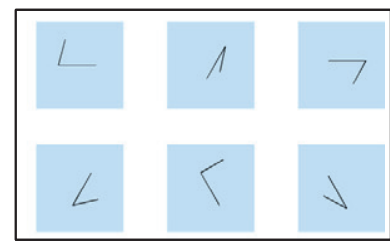

sense, size interference



(C) Interference effect

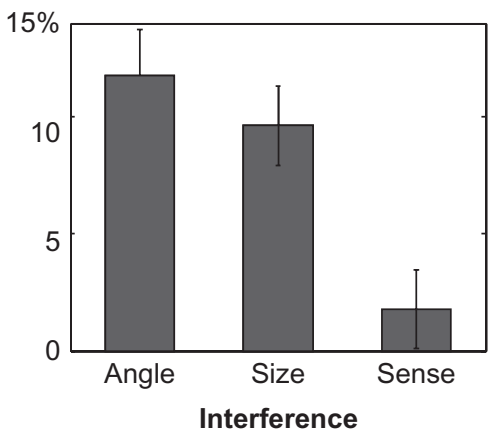

FIGURE 19.2 Test on the perception of angle, size and sense. Participants (US children and adults: $n=104$; Mundurucu children and adults: $n=25$ ) were presented with 27 to 162 slides of six images and asked to pick the image that seemed "different" or "weird and ugly". Trials were of two types. In the "pure" trials (A), the deviant was defined by a single difference in either angle, size, or sense, while the non-deviant figures differed only in orientation. In the "interference" trials, variations were introduced in a second dimension, but not in a way to define a clear deviant. (B) Responses were more accurate to the angle and size pure trials than to the sense pure trials. (C) Also, an interference of angle or size reduced the level of accuracy in the responses, compared to corresponding pure trials without this inference. On the contrary, trials on angle and size were not affected by an irrelevant variation of sense. Adapted from [3]. 
directly, without a need to mentally realign shapes [23]. The context of a Tetris game can facilitate the recruitment of motor resources for children: they can manipulate the shapes with a joystick to fit them in a hole pictured at the bottom of the screen. Our task made it less natural for participants to engage motor resources, because the shapes did not present any motor affordance, and also because the sense trials were interleaved with other trials where mental rotation was not needed. Together, these task features could explain the failure of the youngest participants. More crucially, the judgment we asked from our participants differed from the same/different judgments involved in mental rotation tasks: our instructions (to find a "very different" shape amongst shapes that were "all a little bit different") boiled down to asking participants what type of differences they found relevant to shape classification. The refusal of the children to use mental rotation reveals that sense differences do not appear an important factor of shape classification to their eyes: children did try hard to solve the sense trials, but instead of using mental rotation and checking for sense deviants, they looked at subtle differences in the pixelization, or sometimes chose randomly with evident frustration. These results suggest that adults entertain a more integrated concept of shape, rooted in a variety of cognitive mechanisms, whereas the concept of the children is only linked to shape perception systems. Before the integration of motor resources into the concept of shape, sense does not appear to be an important, defining aspect of shapes, at least in a context where shapes are allowed to vary in orientation.

Together, these results indicate that when classifying shapes, sensitivity to angle and size is universal, while sensitivity to sense is not. When comparing to Euclidean geometry systems, the intuitive geometry implied by the participants' classification of shapes picks the invariants of the non-oriented geometry of solid objects, maybe because these invariants are the most useful in object identification and classification. ${ }^{2}$

\section{NORMATIVE GEOMETRIC CONCEPTS}

We now turn to a second aspect of geometric knowledge, namely the ability to grasp concepts that go beyond any perceptual experience. As a first window into these concepts, we consider first the angle categories. Much as discrete integers are crystallized from a continuous representation of numerosity [24], educated adults crystallize angles into sharp categories of acute, right, and obtuse angles; parallel lines may be viewed as a kind of remarkable angle too. Although it is not necessary to possess a rich conceptual structure to be able to form categories, such absolute, sharp angle categorization is a prerequisite to reasoning about non-perceivable, normative properties, such as the fact that in some special cases, lines may never cross.

We studied children's judgment of categorizations by presenting 141 participants from the US (aged three to 34 years) with displays such as those of Fig. 19.3 [25]. In none of the trials did angle define a clear deviant, unless participants elected to use the angle categories of Euclidean geometry. Indeed, all adults picked the right angles, the parallel lines or the perpendicular lines as deviant in those displays. However, in children, parallelism and right angles were dissociated: all the groups chose the parallel lines as being special, while

\footnotetext{
${ }^{2}$ Nevertheless, the role of metric properties in object identification has been debated: perhaps, the identification of shapes is computed only on the basis of structural or "non-accidental" properties [27,28].
} 


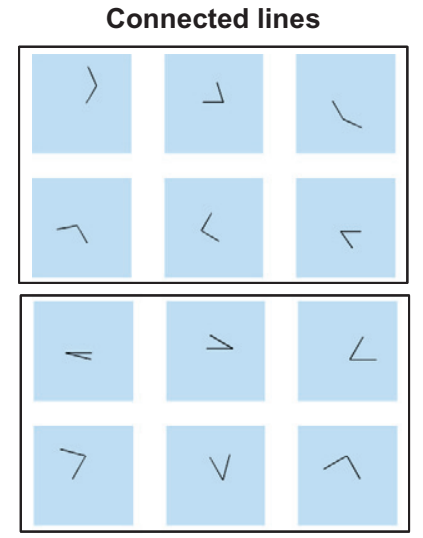

Right angle
Non-connected lines

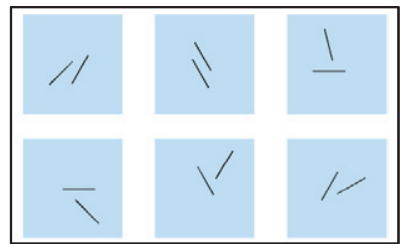

Parallel lines



Perpendicular lines

FIGURE 19.3 Test on angle categories. In this new version of the intruder task, variations in angle do not define a clear deviant unless the participants are sensitive to exact angle categories. Stimuli with connected lines probed the sensitivity to right angle, while stimuli with non-connected lines tested parallel and perpendicular lines.

only children aged seven years and over picked the right angle and perpendicular lines. Furthermore, in a short post-test we asked the children to draw a right angle, parallel lines and perpendicular lines in order to assess their knowledge of the relevant lexical terms: in accord with the general performance at the categorization task, the knowledge of the lexical terms "right angle" or "perpendicular lines" facilitated the detection of these figures in children, while the knowledge of the term for "parallel lines" had no effect on the detection of parallels. These results indicate that different normative categories develop along different trajectories, with a category for parallelism possibly present from start, while the category of right angle gets constructed once the child has acquired the relevant lexicon.

Because some Mundurucu do not receive any instruction in geometry, testing them helps to probe the universality of the right angle category. If specific instruction or an appropriate lexicon is necessary, Mundurucu should not pick a right angle as being special amongst a range of angles; however, if the lexicon just acts as a mere catalyst in the US children, the Mundurucu may direct their choices towards the right angles. Although the previous test has not yet been administered to Mundurucu participants, an examination of a subtest of the trials in the previous experiment (trials on sense with angle interference) could address this question (Fig. 19.2A): indeed, since Mundurucu were not sensitive to sense, these trials must have appeared as a test of angle categories to the participants. Examination of the responses in those particular trials revealed that the Mundurucu did choose the right angles more often than chance. This result raises the possibility that right angle may be a universal category of angle, even though instruction on the relevant lexicon appears to play a role in the acquisition of this category in young US children.

In further research, it would be interesting to evaluate the role of experience in the categorization of right angles by separating different subgroups of Mundurucu participants, 
living either in carpentered villages (where right angles are prominent) or in very rural areas. On the other hand, the performance of the Mundurucu suggests that the role played by instruction in US children may be limited in scope. It is possible that the acquisition of geometric vocabulary helps children forming a category of right angle, without implying any sensitivity to the geometric properties of this figure: perhaps children considered right angles to be special only because they have a name. In this case, the effect of elementary instruction would be initially superficial but may still have enduring effects on the development of geometry, by directing the attention of the child towards categories relevant to Euclidean geometry and therefore facilitating later conceptual learning.

In general, although sharp categories can provide a window onto normative concepts, being able to categorize parallel lines or right angles does not require an elaborate conceptual apparatus and could even be derived from experience. Thus, we attempted to design one last test probing the most abstract geometric concepts directly [26]. Participants were introduced to an ideal shape, either an infinite plane or a sphere. The experimenter narrated the properties of the shape ("it is very, very flat and goes on forever and ever" or "it is very round, like a ball"), straight, infinite lines, as well as dots (Fig. 19.4A). Following this introduction phase, participants were given a list of questions pertaining to the properties of straight lines. Impressively, Mundurucu adults and children performed extremely accurately at the test, especially on the plane. Most of them agreed that a new straight line may always be placed in such way that "it would never cross" a first straight line. They also correctly modulated their responses to adapt them to the planar and spherical environments tested. Beyond categorization, this last test argues for elaborate, non-perceivable concepts being universal.

These concepts may either be part of an innate "core knowledge", or may have been acquired by interactions with the environment. In order to separate these hypotheses, we tested five- and six-year-old US children on the same questionnaire task. Although the young children performed above chance in the plane test, they were much less accurate compared to the other groups; in particular, they responded at chance when asked about parallelism. Moreover, they failed to adapt their responses to the spherical context. These results indicate that the concepts of Euclidean geometry are only partially in place at the age of five or six years; while being universal, Euclidean geometry nevertheless appears to result from a mental construction.

The finding on parallelism has important implications for our interpretation of the results of the previous task. Contrary to adults, the categorization of parallel lines by young children does not rely on a rich conceptual theory of geometry, but probably on perceptual properties of parallel lines, such as the fact that the distance between them is constant, the fact that the two parallel segments look identical, or the fact that parallelism represents a singular point in angle values. Indeed, parallel lines may be regarded as a case where there is no angle to compute, therefore different from all other configurations; or the parallel lines may be perceived as related by an angle of $0^{\circ} / 180^{\circ}$, which is at the same time both a global minimum and a global maximum for non-oriented angles. This singularity might be extracted on the basis of accumulated perceptual experience. Again, as in the case of the category of right angle, the perceptual categorization of parallel lines is compatible with the concepts of geometry. Even if it is not rooted in a rich conceptual understanding of geometry, it could provide a stepping stone to conceptual development. 
(A) Excerpts of the introductory description of the planar and spherical worlds

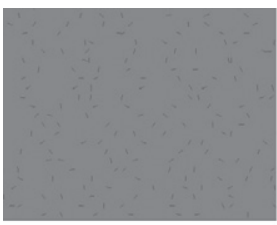

This is a place where the land is flat. It goes on forever.

This is a place where the land is round, like a ball.

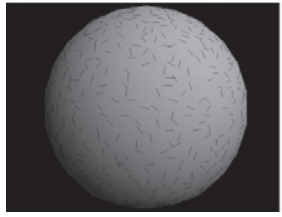



There are paths. Paths are all straight. Paths go straight, always in front of them.

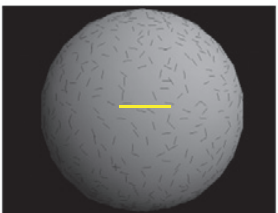

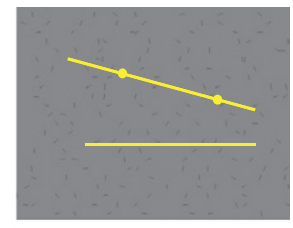

There are villages. All villages are very small. Paths go right through the center of the villages.

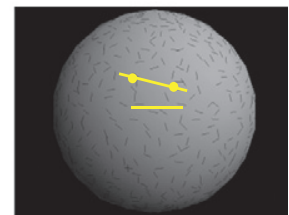

(B) Questionnaire
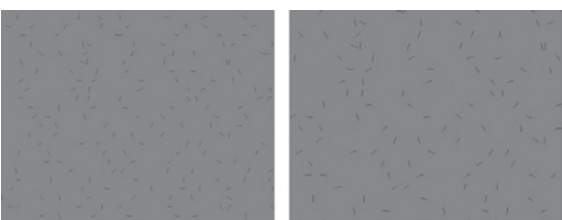

Let us approach to see better... (Zoom)
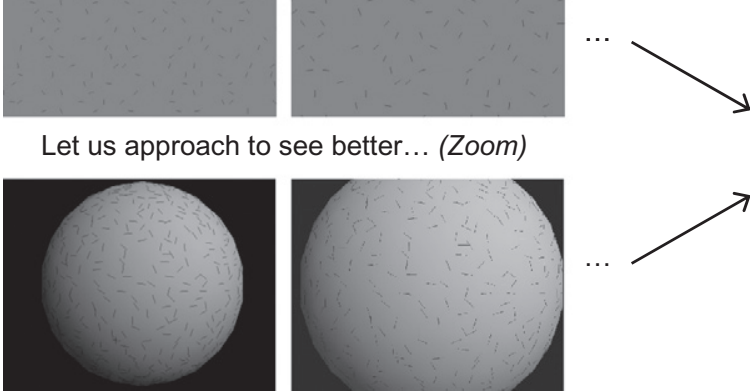

Example of question



Can you place a path that does not cross any of those paths?

FIGURE 19.4 Test of Euclidean reasoning. (A) Participants were first introduced to one of two ideal worlds, shaped either as a plane or a sphere. The experimenter narrated the properties of this world, and introduced the existence of very small villages (points), and paths that never turned or ended (straight lines). Following this introduction, participants were asked a series of 21 illustrated questions pertaining to the properties of points and lines in these worlds (B). Crucially, the sketches presented in the two subsets were identical: a zooming animation created the impression of coming so close to the sphere that it appeared flat.

\section{CONCLUSION}

Our research provides evidence that the basic principles of Euclidean geometry are reflected in intuitions of space that develop progressively throughout childhood, but still appear universal. From early childhood, the perception of shapes provides an intuitive ground corresponding to the geometry of non-oriented solid objects: preschoolers are sensitive to angle and length, can abstract differences of orientation and position, but they also abstract away sense relations. These early intuitions are enriched over development in 
three ways. First, in their classification of shapes, adults recruit a special process to detect sense: mental rotation. Second, mental continua are progressively carved out into discrete categories that give prominence to particular figures. Third, children become able to reason about elaborate non-perceptible concepts. Although the acquisition of the relevant geometric lexicon seems to play a role in the formation of some discrete geometric categories for US children, this role may be reduced to that of a category-maker and catalyst. Indeed, in a population that did not receive any education or share any relevant lexicon in geometry, a brief description of ideal shapes sufficed to elicit elaborate thoughts about the fundamental ideal concepts of Euclidean geometry, such as infinite lines or parallelism.

How could geometry be universal, and at the same time develop progressively? A first possibility would be that geometric knowledge is derived from a type of spatial experience that is so general that every human would encounter it. For example, all humans may come to compute some geometric features of shapes such as angle, or length, because they are especially relevant to object identification and classification [15], or to action. This view raises the question of the development of ideal geometric concepts, which by definition can never be experienced directly, and would need to be derived from incomplete or approximate experience. For example, the Euclidean concept of a line with no width would need to be derived from experience with very thin lines; or the concept of parallelism for infinite straight lines would need to be derived from experience with lines that do not cross locally_still a far cry from the property that parallel lines will never cross. Assuming this derivation to be impossible, Kant famously argued for knowledge of space (as well as geometry) being available a priori to experience [29,30].

Another possibility would be for geometry to be grounded on "core knowledge" [31], i.e. representations of abstract content that were selected by evolution, and provide useful guidelines to interpret the environment and learn. Representations of ideal straight lines or planes may be present in the architecture of the perceptual system to serve as anchors for perception, a strategy that has proven useful to reduce processing loads and increase reliability in artificial vision [32]. Under this hypothesis, during childhood these implicit anchors would need to be progressively reformatted into explicit representations, to be able to enter thought processes and be manipulated directly-a process that may be time consuming, but potentially accessible to every human being.

In conclusion, we have provided evidence that geometric knowledge appears universal, based on two cultures that are maximally different in terms of education and lifestyle. Ultimately, however, claims of universality can never be verified directly, and would be better informed by looking at the factors that favor the acquisition of geometric knowledge. Geometric concepts may emerge spontaneously on the basis of a universal experience with space, or reflect intrinsic properties of the human mind. Precisely what type of experience is relevant, and what limits younger children in their conception of geometry awaits further research.

\section{GLOSSARY}

\section{Universal}

Present in all normally developing human beings, irrespective of their environment, level of education, etc. Some properties may even be universal beyond the human species, but in the present article we will only consider claims pertaining to humans. 


\section{Innate}

Determined by genetic or epigenetic mechanisms, rather than learned from the environment. Some innate features may not be present at birth, as for example the beard in human males. Claims of innateness are hardly accessible to experimentation, contrary to claims of universality.

\section{Intuition}

A form of knowledge that is accessible to explicit report, although its justification is not. In the present experiments, participants were often able to pick the correct response without being able to explain why they took such choice.

\section{Sense}

The geometric property that distinguishes two figures that are mirror images of each other. More generally, given a trajectory in a geometric space, a value of sense can be attributed to this trajectory depending on whether it bears more often to the left or to the right.

\section{Acknowledgments}

The authors thank the members of the "Development Perception-Action" team at the Laboratoire Psychologie de la Perception, the members of E. Spelke's laboratory, two anonymous reviewers and T.R. Virgil for useful discussions and comments on earlier versions of the paper. The work with the Mundurucu is part of a larger project on the nature of quantification. It is based on psychological experiments and linguistics studies conducted in the Mundurucu territory (Pará, Brazil) under the direction of Pierre Pica, in accordance with the Consehlo de Desenvolvimento Cientifico et Tecnologicico and the Fundacão do Indio (Funaï; Processo 2857/04). This work benefited greatly from advice from Lucia Braga (SARAH Network of Neurorehabilitation Hospitals, Brasilia), Andre Ramos (Coordenação Geral de Educação, Funaï), and C. Romeiro (Nucleo de Documentação e Pesquisa, Funaï); M. Karu, and C. Tawe assisted in the data collection. Most of the US children were tested by Danielle Hinchey, Amy Heberle, and Annie Douglas at the Boston Museum of Science, as part of a program coordinated by Marta Biarnes (Boston Museum Science). Supported by INSERM (Stanislas Dehaene), the Département des Sciences Humaines et Sociales of CNRS (Pierre Pica), NIH (Elizabeth Spelke), NSF (Elizabeth Spelke), and the McDonnell Foundation (Stanislas Dehaene).

\section{References}

[1] T.L. Heath, The Thirteen Books of Euclid's Elements, Dover Publications.

[2] S. Dehaene, Core knowledge of geometry in an Amazonian indigene group, Science 311 (2006) 381-384.

[3] V. Izard, E.S. Spelke, Development of sensitivity to geometry in visual forms, Hum. Evol. 23 (2009) $213-248$.

[4] C.R. Gallistel, The Organisation of Learning, MIT Press.

[5] M. Piazza, V. Izard, How humans count: numerosity and the parietal cortex, Neuroscientist 15 (2009) $261-273$.

[6] K. Grill-Spector, R. Malach, The human visual cortex, Annu. Rev. Neurosci. 27 (2004) 649-677.

[7] R.N. Shepard, J. Metzler, Mental rotation of three-dimensional objects, Science 171 (1971) 701-703. 
[8] J.F. Norman, J.T. Todd, V.J. Perotti, J.S. Tittle, The visual perception of three-dimensional length, J. Exp. Psychol., Hum. Percept. Perform. 22 (1996) 173-186.

[9] D. Regan, Evidence for a neural mechanism that encodes angles, Vision Res. 36 (1996) 323-330.

[10] S. Chen, D.M. Levi, Angle judgment: is the whole the sum of its parts?, Vision Res. 36 (1996) 1721-1735.

[11] G.J. Kennedy, Global shape versus local feature: an angle illusion, Vision Res. 48 (2008) 1281-1289.

[12] S.F. Lourenco, J. Huttenlocher, The representation of geometric cues in infancy, Infancy 13 (2008) $103-127$.

[13] A. Slater, Size constancy at birth: newborn infants' responses to retinal and real size, J. Exp. Child Psychol. 49 (1990) 314-322.

[14] A. Slater, Form perception at birth: Cohen and Younger (1984) Revisited, J. Exp. Child Psychol. 51 (1991) 395-406.

[15] E.J. Gibson, A developmental study of the discrimination of letter-like forms, J. Comp. Physiol. Psychol. 55 (1962) 897-906.

[16] A. Shusterman, Young children's spontaneous use of geometry in maps, Dev. Sci. 11 (2008) F1-7.

[17] I. Biederman, E.E. Cooper, Evidence for complete translational and reflectional invariance in visual object priming, Perception 20 (1991) 585-593.

[18] E. Gregory, M. McCloskey, Mirror-image confusions: implications for representation and processing of object orientation, Cognition 116 (2010) 110-129.

[19] J.E. Rollenhagen, C.R. Olson, Mirror-image confusion in single neurons of the macaque inferotemporal cortex, Science 287 (2000) 1506-1508.

[20] S. Dehaene, Reading in the Brain: The Science and Evolution of a Human Invention, Penguin Viking

[21] A. Frick, Motor processes in children's mental rotation, J. Cogn. Dev. 10 (2009) 18-40.

[22] A. Frick, N. Newcombe, Measuring mental rotation in 4-year-olds using a nonverbal touch screen paradigm, in: VI Biennal Meeting of the Congitive Development Society, 2009.

[23] J. Vanrie, Multiple routes to object matching from different viewpoints: mental rotation versus invariant features, Perception 30 (2001) 1047-1056.

[24] S. Dehaene, The Number Sense, Oxford University Press, Penguin Press

[25] V. Izard, D. Hinchey, E.S. Spelke, The development of angle categories, (in preparation).

[26] V. Izard, P. Pica, E.S. Spelke, S. Dehaene, Flexible intuitions of Euclidean geometry in an Amazonian indigene group, (under revision).

[27] I. Biederman, Recognition-by-components: a theory of human image understanding, Psychol. Rev. 94 (1987) 115-147.

[28] I. Biederman, Representation of shape in individuals from a culture with minimal exposure to regular, simple artifacts: sensitivity to nonaccidental versus metric properties, Psychol. Sci. 20 (2009) 1437-1442.

[29] L. Shabel, Reflections on Kant's concept (and Intuition) of space, Stud. Hist. Philos. Sci. 34 (2003).

[30] G. Hatfield, The Natural and the Normative: Theories of Spatial Perception from Kant to Helmholtz, The MIT Press

[31] E.S. Spelke, Beyond core knowledge: natural geometry, Cogn. Sci. 34 (2010) 863-884.

[32] A.P. Gee, Discovering Higher Level Structure in Visual SLAM, Institute of Electrical and Electronics Engineers

[33] E.C. Tolman, Cognitive maps in rats and men, Psychol. Rev. 55 (1948) 189-208.

[34] J. O'Keefe, L. Nadel, The Hippocampus as a Cognitive Map, Clarendon Press, Oxford University Press

[35] K. Cheng, N.S. Newcombe, Is there a geometric module for spatial orientation? Squaring theory and evidence, Psychon. Bull. Rev. 12 (2005) 1-23.

[36] A. Shusterman, E.S. Spelke, Language and the development of spatial reasoning, in: P. Carruthers, et al. (Ed.), The Innate Mind: Structure and Contents, Oxford University Press, pp. 89-106.

[37] N.S. Newcombe, Is cognitive modularity necessary in an evolutionary account of development, in: L. Tommasi, et al. (Ed.), Cognitive Biology: Evolutionary and Developmental Perspectives on Mind, Brain and Behavior, The MIT Press, pp. 105-126.

[38] A. Hupbach, L. Nadel, Reorientation in a rhombic environment: no evidence for an encapsulated geometric module, Cogn. Dev. 20 (2005) 279-302.

[39] S.A. Lee, E.S. Spelke, Signature limits on children's spatial representations: young children navigate by distance and direction but not angle, J. Exp. Psychol., General (in review).

[40] S.A. Lee, E.S. Spelke, Children's use of geometry for reorientation, Dev. Sci. 11 (2008) 743-749.

[41] N.S. Newcombe, J. Huttenlocher, Making Space: The Development of Spatial Representation and Reasoning, The MIT Press

[42] F.C. Klein, A comparative review of recent researches in geometry, Bulletin of the New York Mathematical Society 2 (1893) 215-249. 